\title{
Energy-efficient topology control for wireless sensor networks using online battery monitoring
}

\author{
C. Behrens, O. Bischoff, M. Lueders, and R. Laur \\ Institute for Electromagnetic Theory and Microelectronics (ITEM), University of Bremen, Bremen, Germany
}

\begin{abstract}
This paper presents a first approach towards cooperative energy management in wireless sensor networks using online battery monitoring. Using a standard platform for wireless sensor networks, a simple concept for measuring the battery voltage on the mote with little additional hardware is shown. This is used for modelling of the battery discharge behaviour of Li-Ion batteries and deriving the remaining lifetime under given environmental conditions. As an application example this model can be incorporated into a topology control scheme for optimized network lifetime under variable environmental conditions.
\end{abstract}

\section{Introduction}

Wirelessly networked embedded sensor devices or wireless Sensor Networks (WSN) offer a broad range of applications, from industrial surveillance over security to medical monitoring. Another emerging application of WSN systems is the monitoring of perishable or sensitive freight in logistics. The information provided may be further used in the concept of Autonomous Logistics currently investigated within the Collaborative Research Center 637 at the University of Bremen. This is the main application addressed by the system presented in this paper.

Energy is a scarce resource for this class of devices. Mostly running on batteries as energy source the improvement of energy-efficiency and power management are becoming ever important research topics. Considering common application profiles for Wireless Sensor Networks (WSN), these systems should maintain unattended operation for months, possibly years without being serviced.

Energy efficiency and power management is usually characterized before deployment using network simulators or testbeds. Using this methodology network protocols are tuned to reach the pre-planned power consumption behaviour and are deployed to the real-world afterwards. But this gives

Correspondence to: C. Behrens

(behrens@item.uni-bremen.de) only good results as long the sensor network is operated under almost static environmental conditions because energy models in simulators or testbeds usually do not imply variable environmental conditions.

But real-world application of WSN usually involves these changing conditions. One example of such conditions, especially variable temperature, is found within logistics for monitoring of sensitive or perishable freight. Especially temperature strongly affects the battery behaviour. A model for the battery behaviour should only rely on few input parameters (e.g. battery voltage) and should be computationally simple to be integrated into networking protocols without imposing much overhead. This awareness about their remaining energy level for autonomous wireless devices enables these devices to cooperate on routing of data, aggregation of data and distribution of tasks within the network while allowing for a long network lifetime and a step towards truly adaptive protocols.

The organisation of this paper is shown in the following. In the second chapter the energy consumption of all subsystems of a wireless sensor node is shown and common energy sources for WSN systems are given. The third chapter addresses the problem of online battery monitoring. A measurement circuitry that is based on a common hardware platform for WSN systems is shown. Based on this setup measurements were performed which lead to a simple linear approximation of the temperature behaviour of the applied battery type. As an application example this model may be used in a topology control algorithm for wireless sensor networks which is demonstrated in chapter four. The paper is concluded in chapter five with a summary of the work done and an outlook on future work.

\section{Architecture for Wireless Sensor Network systems}

The basic elements of Wireless Sensor Networks are the network nodes or mainly referred to as "motes". These motes consist of an RF-transceiver, a microcontroller for protocol processing and sensor interfacing, sensors for measuring

Published by Copernicus Publications on behalf of the URSI Landesausschuss in der Bundesrepublik Deutschland e.V. 
Li-lon Battery

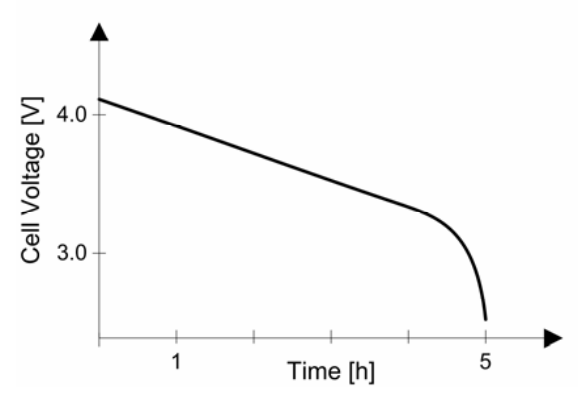

$\mathrm{Ni}-\mathrm{Cd} / \mathrm{Ni}-\mathrm{MH}$ Battery

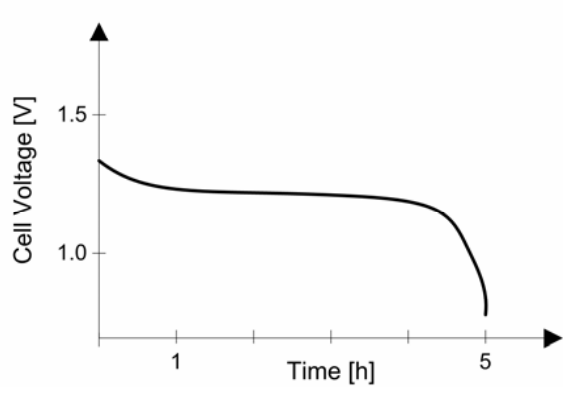

Fig. 1. Typical Li-Ion and NiCd/NiMH battery behaviour.

Table 1. Comparison of power consumption for sending of messages at differing RF power level settings and reading of an internal temperature sensor.

\begin{tabular}{llll}
\hline & $\begin{array}{l}\text { Current } \\
{[\mathrm{mA}]}\end{array}$ & $\begin{array}{l}\text { Duration } \\
{[\mathrm{ms}]}\end{array}$ & $\begin{array}{l}\text { Energy } \\
{[\mu \mathrm{J}]}\end{array}$ \\
\hline Sending of Data Packet (@PA_LEVEL = 3) & 11.16 & 1.82 & 48.75 \\
Sending of Data Packet (@PA_LEVEL = 31) & 22.5 & 1.82 & 98.28 \\
Reading internal temperature sensor & 1.1 & 0.013 & 0.0358 \\
\hline
\end{tabular}

environmental parameters and an energy source which is usually a battery.

\subsection{Energy consumption of WSN components}

We use a hardware platform based on the commercially available TmoteSky system by Moteiv Corporation. These systems use the IEEE 802.15.4-compliant RFtransceiver CC2420 and the 16-bit low-power microcontroller MSP430F1611, both from Texas Instruments. The following measurements were made for the sending of a data message (41 bytes) and the reading of the internal temperature sensor in the microcontroller. The results are shown in Table 1. From these results it may be concluded that adaptively tuning the output power of the transceiver and reducing communication overhead may drastically improve energy efficiency of a single mote and by this increase network lifetime.

\subsection{Battery technologies for WSN systems}

Batteries are the common energy sources for motes. Although energy harvesting may be an option for certain application where there is enough energy (e.g. movement, vibrations, light) in the surroundings of the WSN system. Considering logistics as an application the amount of energy being harvested is much too low to power a mote sufficiently.

In our system we use secondary batteries although the energy density of this type is much smaller compared to primary Zinc-Air or Lithium cells. But the advantage of recharge ability outperforms this drawback.

When considering the discharge curves of the most common types of secondary cells, NiCd and NiMH cells behave almost equally with almost flat discharge behaviour while LiIon batteries have a constant negative slope (see Fig. 1).

This feature of the Li-Ion cell's discharge characteristic offers the advantage of computing the remaining capacity of the battery when the actual load and the temperature of the battery are known. We consider Li-Ion batteries as being more favourable then using Ni-based batteries because the small slopes of Ni-based batteries impose higher demands on the measurement of the voltage as small changes in output voltage result in larger changes in discharge capacity. Therefore, Li-Ion cells were selected due to their higher energy density and their behaviour towards discharge modelling. As a voltage regulator is used in our mote, the higher slope of the output voltage does not affect the performance of the mote itself.

\section{Battery monitoring}

As discussed in the previous chapter, Li-Ion batteries are more favourable for modelling due to their higher negative slope. This enables the usage of the internal AD-converter of microcontroller of the mote. It offers a sufficient resolution of 12 bits for the measurement of the battery voltage.

\subsection{Circuitry and measurements}

The measurement setup requires minimum additional hardware for the mote system. (see Fig. 2). In order to verify this setup a series of measurements was taken under controlled temperature using a constant load. As battery we selected the Panasonic CGA103450A cell with a nominal output voltage of $3.6 \mathrm{~V}$. The results for $10^{\circ} \mathrm{C}$ and $-10^{\circ} \mathrm{C}$ are also shown in Fig. 2 and reflect the temperature dependency of the discharge behaviour. 

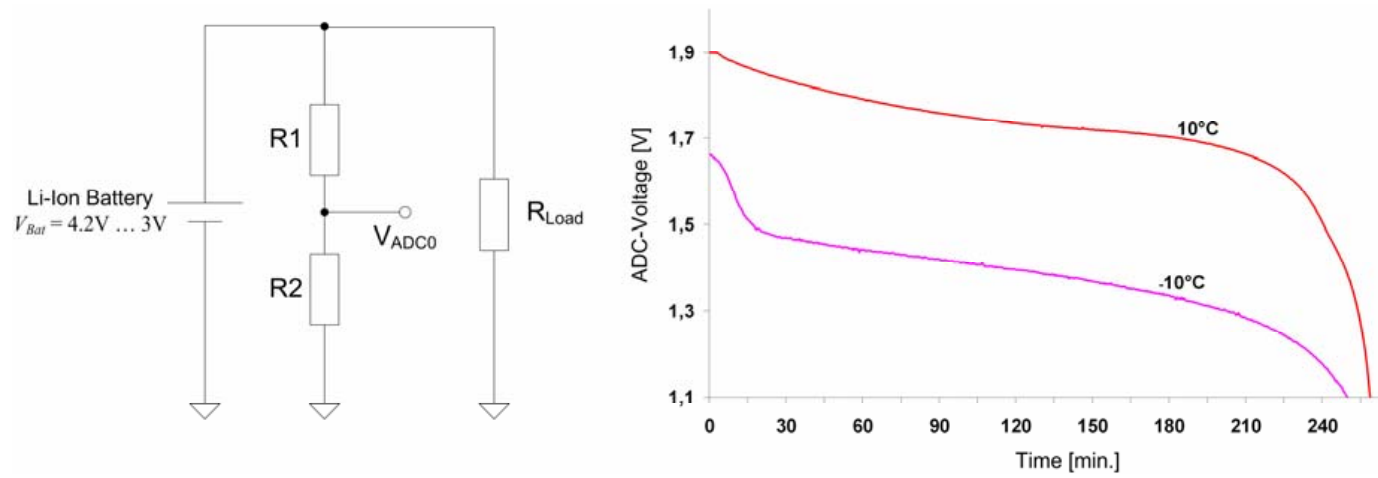

Fig. 2. Measurement setup and resulting graphs for $-10^{\circ} \mathrm{C}$ and $10^{\circ} \mathrm{C}$.

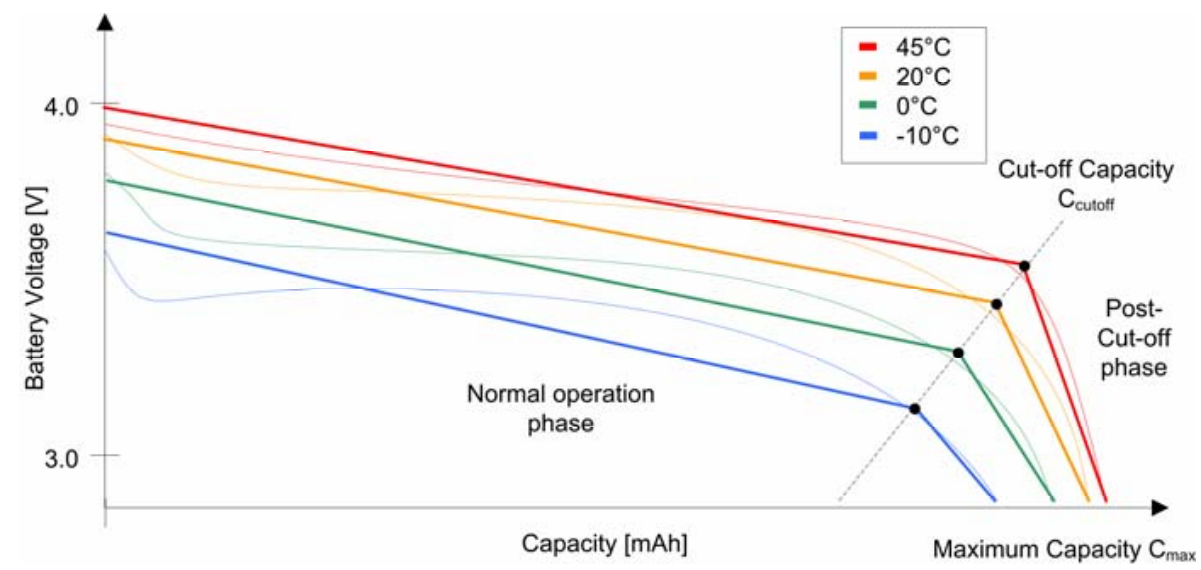

Fig. 3. Approximated discharge characteristics with varying temperature.

\subsection{Modelling}

Using these measurements, a simple numeric model was generated by dividing the discharge curve into two phases: normal operation and post-cut-off. The normal operation is given for the slightly decreasing slope. Post-cut-off is given just before the battery is empty. Both phases can be described by a simple linear model as a set of four numerical parameters $c_{0}$ to $c_{3}$, the temperature $\vartheta$ and the output voltage of the battery $V_{\text {out }}$ in the following equation:

$C=\left(c_{0}+c_{1} \cdot \vartheta\right)+\left(c_{2}+c_{3} \cdot \vartheta\right) \cdot V_{\text {out }}$.

Furthermore a cut-off capacity is defined which clearly marks the border of the two operational phases:

$C_{\text {cutoff }}=C_{\text {base }}+c_{4} \cdot \vartheta$

$C_{\text {base }}$ is a numerically found base capacity and constant $c_{4}$ is the slope of the cut-off capacity with varying temperature $\vartheta$. The switch-over between these phases is realized by using a different parameter set ( $c_{0}$ to $c_{3}$ ) for Eq. (1). The maximally usable capacity can be calculated by defining an end-point voltage which is used in adding Eqs. (2) and (1) with the cut-off parameters. This endpoint voltage is e.g. dependent on the used voltage regulator type and the minimum system supply voltage. By simply subtracting the current capacity from this maximum capacity, the residual capacity is calculated. The resulting graph is shown in Fig. 3.

Other approaches to battery modelling use much more complex models (e.g. Benini's discrete-time model) which were originally developed for mobile PC platforms. Due to their complexity these models are not suitable for mote-type systems as they impose too much overhead.

\section{Application in topology control}

To benefit from the battery information and prolong the network lifetime we introduce a sample application controlling the topology of a sensor network. In a shared-channel wireless sensor networks the high density of motes entails to a network redundancy so that already a small contingent of motes is adequate to form the multi-hop spanning tree and affords data forwarding. A Cluster-based Energy Conservation (CEC) Algorithm does not rely on location information, but avails of useful connectivity characteristics, e.g. link quality, 


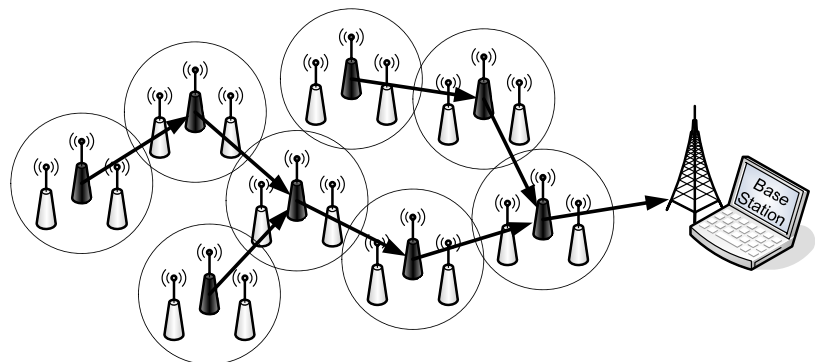

Fig. 4. Spanning tree of a cluster-based wireless sensor network.

neighbour proximity and/or residual energy, to minimize network redundancy and therewith energy consumption.

In a cluster-based network, the cluster-head nodes have increased energy consumption since they construct the backbone of the network. Therefore, the accuracy of the energy consumption model has to be very precise to ensure equitable load distribution. The iHEED clustering protocol provides a Credit-Point-System (CREP) to characterize the energy consumption of each packet transmission.

The mote is initialized with a maximum residual energy, which will be progressively decreased during every transmission. In equidistant intervals, the clustering process is triggered to classify the motes in cluster-heads and clustermembers. Cluster-heads raise their transmission power for inter-cluster communication to forward data across neighbouring cluster-heads via multi-hop toward the base-station. For intra-cluster communication, cluster-members decrease the transmission power and send data to their cluster-heads via single-hop (see Fig. 4).

The main parameter of the cluster election is the residual energy of the mote. Thus, it is necessary to improve the estimation of the residual energy and thereby prolong the network lifetime. A periodically recursive adaptation of the residual energy using online battery monitoring would be an innovative enhancement for wireless sensor networks under dynamic environmental conditions.

\section{Conclusions}

The need for information on residual energy in wireless sensor networks is an emerging field of research. The temperature behaviour of batteries may strongly affect the residual energy and thereby the performance of the network. Based on a standard platform, a simple measurement setup and a computationally simple linear model was developed which is capable of calculating the residual energy as a function of temperature directly on the mote. This information may be used in power-adaptive protocols, e.g. the routing and topology control protocol set iHEED.

For future work the performance of this model will be evaluated by deploying battery model based algorithms in a testbed. The model will be enhanced by the inclusion of other effects on battery performance (e.g. self-discharge, load-dependence). Further optimization of the model will also be considered in the near future.

Acknowledgements. This research was supported by the German Research Foundation (DFG) as part of the Collaborative Research Centre 637 "Autonomous Cooperating Logistic Processes".

\section{References}

Chen, B., Jamieson, K., Balakrishnan, H., and Morris, R.: SPAN: An Energy-Efficient Coordination Algorithm for Topology Maintenance in Ad Hoc Wireless Networks, Mobile Computing and Networking Conference, 85-96, 2001.

Gehrke, J. D., Behrens, C., Jedermann, R., and Morales Kluge, E.: The intelligent Container - Toward Autonomous Logistic Processes, in: KI 2006 Demo Presentations, University of Bremen, 15-18, 2006.

Milenkovic, A., Milenkovic, M., Jovanov, E., Hite, D., and Raskovic, D.: An environment for runtime power monitoring of wireless sensor network platforms, Proc. of SSST'05, 406-410, 2005.

Panasonic CGA103450A Li-Ion cell datasheet: http: //www.panasonic.com/industrial/battery/oem/images/pdf/ Panasonic_LiIon_CGA103450A.pdf, 2006.

Rao, R. and Rakhmatov, D. N.: Battery Modeling for Energy-Aware System Design, IEEE Computer, 36(12), 77-87, 2003.

Shnayder, V., Hempstead, M., Chen, B., Allen, G. W., and Welsh, M.: Simulating the power consumption of large-scale sensor network applications, Proc. 2nd international Conference on Embedded Networked Sensor Systems, ACM Press, New York, NY, 188-200, 2004.

Texas Instruments MSP430F1611 Microcontroller Datasheet: http: //focus.ti.com/lit/ds/symlink/msp430f1611.pdf, 2006.

TMoteSky Datasheet, Moteiv Cooperation: http://www.moteiv. com/products/docs/tmote-sky-datasheet.pdf, 2006.

$\mathrm{Xu}$, Y., Bien, S., Mori, Y., Heidemann, J., and Estrin, D.: Topology Control Protocols to Conserve Energy in Wireless Ad Hoc Networks, CENS Technical Report 0006, 2003.

Younis, O. and Fahmy, S.: An experimental study of routing and data aggregation in sensor networks, Conference on Mobile Ad hoc and Sensor System, 8-15, 2005. 